\title{
Investigating the relationship between fetus heart rate in the first trimester screening with fetus sex
}

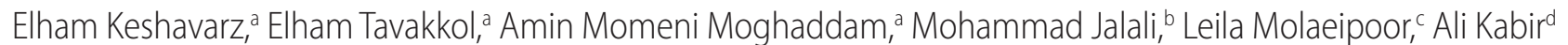

aDepartment of Radiology, Mahdieh Hospital, Shahid Beheshti University of Medical Sciences, Tehran, Iran.

bDepartment of Cardiology, School of Medicine, Alborz University of Medical Sciences, Karaj, Iran.

'Department of Epidemiology, Pasteur Institute of Iran, Tehran, Iran.

'Minimally Invasive Surgery Research Center, Iran University of Medical Sciences, Tehran, Iran.

Correspondence to Ali Kabir (email: aikabir@yahoo.com).

(Submitted: 17 August 2017 - Revised version received: 05 September 2017 - Accepted: 13 November 2017 - Published online: 26 March 2018)

\begin{abstract}
Objective There is a common belief that sex of the fetus is predictable by assessing fetal heart rate (FHR) in the early weeks of pregnancy. We aimed to assess the relationship between FHR in the first trimester screening with fetus sex.

Methods This mixed prospective-retrospective cohort study is done on 374 fetus samples of pregnant women who referred to evaluate nuchal translucency (NT) in a hospital in Tehran, Iran between October 2013 and March 2015. Adjusted multivariable linear regression model, receiver operating characteristic (ROC) curve and Youden index were used in analysis.

Results There were $52.9 \%$ male among fetuses. The median and interquartile range (IQR) of FHR in male and female fetus were 155.5 $(152-160)$ and $155(150-160)$ beats per minute $(\mathrm{bpm})$, respectively $(P=0.213)$. The crude regression results showed that the average FHR of the male fetuses was $1.27 \mathrm{bpm}$ higher than females but it was not statistically significant $(P=0.131)$. The adjusted regression results showed that fetus sex was not significantly associated with FHR in terms of similar age and mother's gravid and parity. Area under the ROC curve was 0.54 (95\% confidence interval: $0.48,0.59$ ) which shows very low FHR power to determine sex of the fetus in the first trimester of pregnancy. The best cut off point was equal to 152 pulse bpm, sensitivity and specificity were 82.81 and 30.11 , respectively.

Conclusion The results showed that FHR is not a good factor to determine sex of the fetus.

Keywords fetal, first, heart rate, maternal age, pregnancy trimester, sex, ultrasonography
\end{abstract}

\section{Introduction}

Increasing gestational age, pre-pregnancy weight gain, inactivity and major changes in maternal nutrition are among important factors in increasing high-risk pregnancies in recent decades. Hypertension disorders, obesity and gestational diabetes are among important risk factors that affect health of the fetus and newborn in addition to maternal health threatening.

Studies in recent decades have shown that the fetus sex during pregnancy is associated with pregnancy and childbirth complications. ${ }^{2}$ A male fetus is exposed with higher complication of catching disease and mortality during pregnancy and infant period. ${ }^{3}$ In many studies, male gender is known as an independent risk factor associated with pregnancy and childbirth complications. ${ }^{4,5}$ The male fetus is associated with a higher risk of developing gestational diabetes, preeclampsia during pregnancy, congenital anomalies and X-dependent genetic diseases. ${ }^{4,6,7}$ On the other hand, male sex is accompanied with a higher risk of preterm delivery, prolapsed umbilical cord and placenta previa. ${ }^{5}$ Also the likelihood of cesarean delivery and use of assistive devices for delivery of a male is more than female fetuses. The prevalence of being underweight during birth also has been reported more in male than female infants. ${ }^{4,8}$ Hence, it seems that awareness about sex of the fetus in the early weeks of pregnancy and following diagnostic, treatment and appropriate supportive interventions are effective in improving health of the fetus and newborn. On the other hand, recognizing sex of the fetus in the first trimester of pregnancy, can resolve parents prompt request to know their child's gender and subsequently prepares them a perfect opportunity to provide cloths and other needs.

Various methods are used to detect fetus sex during pregnancies, which are mainly based on ultrasound techniques. Using ultrasound method to determine sex of the fetus in the second and third trimester is highly efficient. However, the diagnostic power of this tool in the first trimester of pregnancy is weak and unreliable. ${ }^{9}$ There is a common belief among pregnant women and some doctors that sex of the fetus in the early weeks of pregnancy is predictable by assessing fetal heart rate (FHR). Based on this view, the girls FHR is more than boys. ${ }^{10}$ However, in previous studies very contradictory results have been reported regarding the predictive power of this method. ${ }^{11,12}$

Till now, few citable studies have been performed around the world and Iran about the predictor role of FHR in determining fetus gender in early pregnancy. ${ }^{12-14}$ Different factors are important to measure FHR that have not been mentioned in most previous studies including how to measure FHR, FHR changes, gestational age, maternal age and mother's gravid and parity. ${ }^{15-17}$ Due to the above-mentioned evidence, it seems that early detection of fetus sex in the first trimester of pregnancy can effectively reduce morbidity and mortality in fetus and newborn by providing supportive measures by doctors and parents. Hence, with applying same conditions to measure FHR and considering possible confounding factors listed above, we tried to evaluate the association between FHR in the first trimester of pregnancy screening with the baby's gender.

\section{Materials and Methods}

This is a mixed prospective-retrospective cohort study. This study is done with the approval of Shahid Beheshti University of Medical Science's Research Council on sample of the fetuses of pregnant women who referred to Mahdieh hospital, which is a third level referral hospital, to evaluate nuchal translucency in Tehran Province, Iran in between October 2013 and March 2015. 
The sample size was estimated 374 fetuses considering type one error $(\alpha)$ equal to 0.01 , mean and standard deviation of FHR $167.3 \pm 10.1$ in boys and $167 \pm 9.1$ in girls, ${ }^{14}$ and by using the formula of comparing the mean of two independent community. But with considering the loss of $20 \%$ of our cases during follow-up, 451 cases were considered. After receiving informed written consent from parents, singleton pregnant mother's FHR who had pregnancy ultrasound between 11 and 14 weeks and 2 days of pregnancy with crown-rump length (CRL) length of 45-84 mm, was measured by M-mode ultrasound (GE E6) and recorded in the first seconds of the exam when the fetus was in rest.

Other fetuses with structural abnormalities, the uncertainty of the exact date of pregnancy, abortion, bleeding, multiple pregnancy, congenital diseases, invalid last menstrual period (LMP), decreased amniotic fluid and uncertain sex of the fetus (with any reason in next referrals) were excluded from the study. Ultrasound results were measured by different people and then to reduce bias between observers, all ultrasound results were reviewed by researchers. Fetus sex was determined through a phone call or in person after the birth. Other required information including week of pregnancy, maternal age, mother's gravid and parity, abortion, bleeding during pregnancy, decreased amniotic fluid, the exact date of pregnancy (valid LMP) and congenital anomalies detected in fetus ultrasound screening were fully extracted from available records using checklist.

To describe the data, descriptive statistics [frequency, median, and interquartile range (IQR)] were used. To investigate the relationship between qualitative and quantitative variables, chi-square, Mann-Whitney and the median test were used. To investigate the role of predictor variables in determining FHR, we used crude and adjusted multivariable linear regression model. Also to eliminate confounding factor's effect, the Backward Stepwise Model was used. In the Backward Stepwise Model by Hosmer and Lemshow method, only variables with $P$-value $<0.2$ were included in final model. For estimating FHR accuracy in predicting fetus sex in the first trimester of pregnancy, the receiver operating characteristic (ROC) curve was drawn. The Youden index was used for detecting the optimal cut off point. Data analysis was done using STATA 11.2 software (STATA Corp. LP) and a confidence interval level of $99 \%$.

\section{Results}

In this study, 451 patients were studied in which 60 cases were excluded for various reasons including abortion, bleeding, multiple pregnancy, congenital diseases and other associated conditions with exclusion criteria. Also, 17 cases were excluded because of uncertainty about the newborn's gender despite our telephone follow-up. The average FHR of 17 eliminated fetuses was not significantly different from those remained in the study $(P=0.316)$. Finally, a total number of 374 fetuses between 11 and 14 weeks and 2 days of pregnancy were enrolled in the study according to the study criteria.

From the total number of 374 samples, 198 cases (52.9\%) were male. The most male and female fetuses were at 12 th and 13th week of pregnancy, respectively. The median (IQR) of FHR in male and female fetuses were 155.5 (152-160) and 155 (150$160)$ beats per minute (bpm), respectively. The average of mother's ages in both male and female sexes was about 24 years with a range of $15-37$ years. More than $70 \%$ of mothers of both boys (74.75\%) and girls $(72.73 \%)$ had one or two previous pregnancy history. Chi-square test showed that variable levels including

Table 1. Demographic and obstetric characteristics in the first trimester screening by fetal gender

\begin{tabular}{|c|c|c|c|c|c|}
\hline \multirow{2}{*}{ Variables } & \multicolumn{2}{|c|}{ Male (Number = 198) } & \multicolumn{2}{|c|}{ Female (Number = 176) } & \multirow{2}{*}{$P$-value } \\
\hline & Number & Percentage & Number & Percentage & \\
\hline Gestational age (week) & & & & & 0.072 \\
\hline 11 & 20 & 10.10 & 17 & 9.66 & \\
\hline 12 & 90 & 45.45 & 90 & 51.14 & \\
\hline 13 & 84 & 42.42 & 58 & 32.95 & \\
\hline 14 & 4 & 2.02 & 11 & 6.25 & \\
\hline Gravidity & & & & & 0.110 \\
\hline 1 & 75 & 37.88 & 57 & 32.39 & \\
\hline 2 & 73 & 36.87 & 71 & 40.34 & \\
\hline 3 & 46 & 23.23 & 48 & 27.27 & \\
\hline 4 & 4 & 2.02 & 0 & 0.00 & \\
\hline Parity & & & & & 0.154 \\
\hline 1 & 108 & 54.55 & 80 & 45.45 & \\
\hline 2 & 70 & 35.35 & 81 & 46.02 & \\
\hline 3 & 20 & 10.10 & 15 & 8.52 & \\
\hline Maternal age (years) & & & & & 0.778 \\
\hline $15-20$ & 39 & 19.70 & 35 & 19.89 & \\
\hline $21-25$ & 80 & 40.40 & 65 & 36.93 & \\
\hline $26-30$ & 55 & 27.78 & 57 & 32.39 & \\
\hline $30<$ & 24 & 12.12 & 19 & 10.80 & \\
\hline
\end{tabular}




\begin{tabular}{|c|c|c|c|c|c|c|}
\hline Variables & $\begin{array}{l}\text { Unadjusted regression } \\
\text { coefficient }\end{array}$ & $\% 95 \mathrm{Cl}$ & $P$-value & $\begin{array}{l}\text { Adjusted regression } \\
\text { coefficient* }\end{array}$ & $\% 95 \mathrm{Cl}$ & $P$-value \\
\hline \multicolumn{7}{|l|}{ Gender } \\
\hline Female & $\operatorname{Ref}^{\#}$ & & & & & \\
\hline Male & 1.27 & $-0.380,2.92$ & 0.131 & & & \\
\hline \multicolumn{7}{|c|}{ Gestational age (week) } \\
\hline 11 & $\operatorname{Ref}^{\#}$ & & & $\operatorname{Ref}^{*}$ & & \\
\hline 12 & -2.05 & $-4.89,0.78$ & 0.156 & -2.46 & $-5.27,0.36$ & 0.087 \\
\hline 13 & -3.70 & $-6.60,0.80$ & 0.013 & -3.84 & $-6.75,-0.93$ & 0.01 \\
\hline \multicolumn{7}{|l|}{ Gravidity } \\
\hline 1 & Ref $^{f}$ & & & Ref* $^{*}$ & & \\
\hline 2 & -0.44 & $-2.33,1.45$ & 0.646 & 0.19 & $-1.71,2.10$ & 0.841 \\
\hline 3 & -1.33 & $-3.43,0.80$ & 0.222 & -0.97 & $-3.07,1.12$ & 0.362 \\
\hline \multicolumn{7}{|l|}{ Parity } \\
\hline 1 & $\operatorname{Ref}^{*}$ & & & & & \\
\hline 2 & 0.34 & $-1.41,2.08$ & 0.703 & & & \\
\hline 3 & 1.58 & $-1.35,4.52$ & 0.290 & & & \\
\hline \multicolumn{7}{|c|}{ Maternal age (years) } \\
\hline $15-20$ & $\operatorname{Ref}^{\#}$ & & & & & \\
\hline $21-25$ & 1.70 & $-0.57,3.98$ & 0.143 & & & \\
\hline $26-30$ & 1.77 & $-0.62,4.16$ & 0.146 & & & \\
\hline $30<$ & 1.46 & $-1.60,4.52$ & 0.347 & & & \\
\hline
\end{tabular}

$\S:$ confidence interval.

weeks of pregnancy, gravid, parity and maternal age had no significant difference between both sexes (Table 1).

Overall, the median FHR showed no significant difference between both sexes $(P=0.579)$. The median test showed that the median of both sex's FHR due to different levels of age, mother's parity and gravidity and weeks of pregnancy had no significant difference.

To predict changes in FHR average, the effect of each variable including sex of the fetus, week of pregnancy, gravid, parity and maternal age were examined using crude linear regression. The result of our analysis showed that the average FHR of male fetuses was 1.27 times per minute higher compared to female fetuses but it was not statistically significant $(P=0.131, \beta=1.27)$. Age, mother's gravid and parity did not show a significant relationship with FHR. Also, the adjusted regression results showed that none of the mother's gravid levels had significant relationship with FHR (gravid 2 and 3 compared to gravid 1 had $\beta=-0.97$ and 0.19 , respectively) (Table 2). Mean FHR of 13th and 14th week of pregnancy was significantly 3.8 and 7.9 beats which was lower than 11 th week, respectively, after adjustment for gravidity $(P=0.01$ and 0.001 , respectively) (Table 2).

Predicting fetus sex in the first trimester of pregnancy by FHR was drawn by ROC curve (Fig. 1). For drawing ROC curve, two vertical and horizontal axes were defined as sensitivity and specificity. Considering Youden index, the best cut off point was equal to 152 pulse bpm (sensitivity of 82.81 and specificity of 30.11). In ROC curve, the AUC is between 0 and 1 and whatever the number is closer to one (slope of the curve is upward and to the left), it indicates the higher strength of the FHR in predicting the baby's gender. Area under the ROC

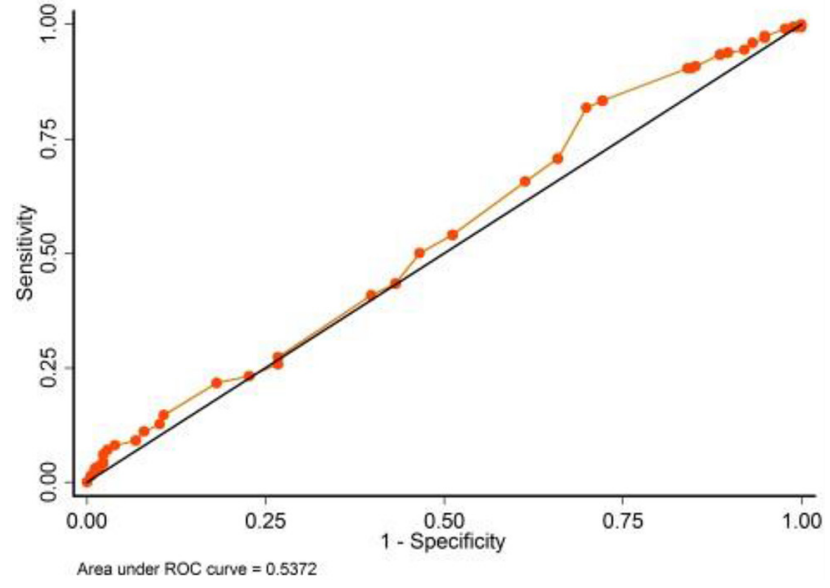

Fig 1. The predicted fetus sex, according to fetal heart rate in the first trimester of pregnancy by using receiver operating characteristic curve.

curve was estimated 0.54 with a confidence interval of 0.48 , 0.59 which shows very low FHR power to determine sex of the fetus in the first trimester of pregnancy. It covers 50\% and shows that the probability of choosing FHR for predicting fetal sex is " $50-50$ " and is not different with chance.

\section{Discussion}

This study investigated the relationship between FHR in the first trimester and fetus sex. We controlled the possible confounding role of FHR changes during different periods of pregnancy in the design phase of this study. To do so, only 
embryos who had minimum amount of FHR fluctuations were studied. In other words, only fetuses between 11 and 14 weeks and 2 days of pregnancy were enrolled in the study. ${ }^{18}$ On the other hand, to eliminate confounding factor effects, such as maternal bleeding, multiple pregnancy, fetal abnormalities and other things, a total of 77 cases were excluded according to exclusion criteria.

To unify data collection tool, the FHR was measured by ultrasound device in a state of immobility and to reduce bias between the observers, ultrasound results were read and checked by the researcher. Also to adjust the gravid, parity and maternal age roles, the adjusted linear regression was used in analyzing data.

Our findings showed that despite moderating many possible factors influencing FHR (mother's parity, gravid and age), any significant correlation was not seen between heart rate in the first trimester of pregnancy with fetal sex. In a study in 1964 on 95 fetuses with weekly measuring of the FHR during the final 2 months of pregnancy, the average of girls and boys FHR was 141.7 and $140.0 \mathrm{bpm}$, respectively. This result is consistent with ours and shows no relationship between FHR with fetal sex. Moreover, this study showed that FHR pattern is different for boys and girls. So that often boy's heart rhythm is based on linear pattern and girls one is more based on complex pattern. So it is possible to determine the sex of the fetus by FHR pattern help. However, it should be noted that this study is associated with FHR in the third trimester of pregnancy and its comparability to the first trimester is less. Other limitations of this study include small sample size. ${ }^{19}$

In other study, FHR of 220 pregnant women at 14-41 weeks of pregnancy was recorded. No significant correlation between FHR and the baby's gender was observed at any stage of pregnancy.11 This study was done in a big range of pregnancy with high FHR fluctuation and people during the first, second and third trimesters of pregnancy were entered into the study. In fact, fluctuations of the FHR were not controlled in this study.

Another study on 477 pregnant women with fetuses under 14 weeks, which sonography was done on them to check factors such as bleeding in the first trimester and uncertainty of the exact date of pregnancy were entered into the study. The sonography samples were repeated at 18-24 weeks by the same team and fetal sex was determined, $51 \%$ were boys. Like our study, average maternal age, gravity, parity and gestational age did not have significant difference between boys and girls. Boys and girls FHR mean was 154.9 \pm 22.8 and $151.7 \pm 22.7 \mathrm{bpm}$, respectively, without significant difference $(P=0.13)$. Among important points in generalization a study, is to select sample in a way that can be the representative of the studied population. McKenna enrolled the people into the study who mainly did sonography due to disease or complication of pregnancy. So it seems that these people are not the representative of healthy mothers and fetuses. While in our study, only mothers who referred for routine screening ultrasound were examined and pregnancies with complications were excluded. ${ }^{12}$ Also these patients entered the study under 14th week of pregnancy and it is possible that FHR be slower in 6-7 weeks of pregnancy compared with 8-14 weeks.20 Our study was conducted at 11th and 14th week of pregnancy. During this period, the minimum FHR fluctuations occur in pregnancy. ${ }^{20}$
One study on 655 fetuses (female: $50.7 \%$ ) in 8-13 weeks of pregnancy showed that the boys and girls FHR mean in the first trimester of pregnancy was $167 \pm 9.1$ and $167.3 \pm 10.1$ bpm $(P=0.62) \cdot{ }^{14}$ In other similar studies, it was shown that contrary to public perception about faster FHR in girls, there was no difference between boys and girls in this point. ${ }^{12,21-23}$

Up to now, the studies done about the relationship between FHR and fetal sex often had very low sample size. ${ }^{19,24,25}$ On the other hand, most of the studies are done in a big range of pregnancy and are often performed in the second and third trimester of pregnancy. ${ }^{11,22,24,26}$ Therefore, fluctuations that occur in FHR in different phases of pregnancy, are not considered in many of the studies. Also, lot of different tools are used to auscultate FHR in previous studies and because the accuracy of diagnostic tools and their power is not evaluated in these studies; thus, the comparison chance between studies is less. ${ }^{14}$ However, this study was done with appropriate sample size and high statistical power and with a short distance from pregnancy in 11-14 weeks and 2 days that in this period FHR has lower fluctuation. ${ }^{12}$ Also, an ultrasound device with high diagnostic power is used. On the other hand, the role of important effective variables, such as maternal age, mother's gravid and parity were adjusted in this study so it seems that the results of this study can be reliable. We were faced with some limitations in this study. Some families refused to cooperate with the researcher to determine sex of the baby in the follow-up period. Also, a limited number of patients' data were collected through telephone calls that may cause recall bias. Despite all these limitations, studying FHR in a period with more uniform situation, adjusting for possible confounding factors are among our strengths. Moreover, although sonographies were done by different people, but all sonographies results were reviewed by the researchers. So the observer bias reduced to its minimum level.

Median of FHR was not different between various weeks of pregnancy. However mean FHR, adjusted for gravidity, had a decreasing trend with increase in gestational week which was not different with 12th and 11th week, but significantly different between 13th or 14th week and 11th week. However, this difference (4 or 8 bpm) was neither big nor clinically significant nor measurable by Doppler sonography. So, despite the common believe that FHR has minimum fluctuations during 11th to 14th gestational week, 20 our study showed that there are some small changes during these weeks after controlling the confounder effect of gravidity.

\section{Conclusion}

The results of this study showed that FHR is not a good predictive factor to determine sex of the fetus. Due to the importance of health point of early fetal sex diagnosis in identifying disorders related to gender especially at the same time with the first trimester screening, it is recommended to pay more attention to that in next studies. And further investigation of this hypothesis should be done with taking into account other unknown variables that may be effective in relation to gender and FHR.

\section{Conflict of Interest}

None. 


\section{References}

1. Bernardes J, Gonçalves H, Ayres-de-Campos D, Rocha AP. Linear and complex heart rate dynamics vary with sex in relation to fetal behavioural states. Early Hum Dev. 2008;84:433-439.

2. Goncalves H, Ayres-de-Campos D, Bernardes J (Eds.). The effect of gender, gestational age and behavioral states on fetal heart rate variability, 8th Conference of the European Study Group on Cardiovascular Oscillations (ESGCO), 2014; IEEE, Trento, Italy.

3. Saadia Z, Farrukh R. Association between fetal sex ratio and maternal eclampsia-A descriptive study in Pakistani population. Internet J Gynecol Obstet. 2009;12.

4. Graatsma M. Monitoring of Fetal Heart Rate and Uterine Activity, 1st ed., Amsterdam; 2010.

5. Odeh M, Grinin V, Kais M, Ophir E, Bornstein J. Sonographic fetal sex determination. Obstet Gynecol Surv. 2009;64:50-57.

6. Ogueh $\mathrm{O}$, Steer P. Gender does not affect fetal heart rate variation. $\mathrm{Br} J$ Obstet Gynaecol. 1998;105:1312-1314.

7. Dawes NW, Dawes GS, Redman CW. Fetal heart rate patterns in term labor vary with sex, gestational age, epidural analgesia, and fetal weight. Am J Obstet Gynecol. 1999;180:181-187.

8. Hanprasertpong T, Phupong V. First trimester embryonic/fetal heart rate in normal pregnant women. Arch Gynecol Obstet. 2006;274:257-260.

9. Druzin ML, Hutson JM, Edersheim TG. Relationship of baseline fetal heart rate to gestational age and fetal sex. Am J Obstet Gynecol. 1986;154:1102-1103.

10. Barcroft J. Fetal circulation and respiration. Physiol Rev. 1936;16:103-128.

11. Genuis SK, Genuis S, Chang WC. Antenatal fetal heart rate and "maternal intuition" as predictors of fetal sex. J Reprod Med. 1996;41:447-449.

12. Bernard J. Prediction from human fetal measures. Child Dev. 1964;35:1243-1248.

13. Aibar L, Puertas A, Valverde M, Carrillo MP, Montoya F. Fetal sex and perinatal outcomes. J Perinat Med. 2012:40:271-276.

14. Sheiner E, Levy A, Katz M, Hershkovitz R, Leron E, Mazor M. Gender does matter in perinatal medicine. Fetal Diagn Ther. 2004;19:366-369.

15. Jimenez DF, Tarantal AF. Fetal gender determination in early first trimester pregnancies of rhesus monkeys (Macaca mulatta) by fluorescent PCR analysis of maternal serum. J Med Primatol. 2003;32:315-319.

16. Bracero LA, Seybold DJ, Witsberger S, Rincon L, Modak A, Baxi LV. First trimester fetal heart rate as a predictor of newborn sex. J Matern Fetal Neonatal Med. 2016;29:803-806.

17. Jones J, Weerakkody Y. Fetal heart rate: TrikeApps 2015 [updated 2015]. Available from: http://radiopaedia.org/articles/fetal-heart-rate.

18. Akbarzade M, Rafiee B, Asadi N, Zare N. The effect of maternal relaxation training on reactivity of non-stress test, basal fetal heart rate, and number of fetal heart accelerations: a randomized controlled trial. Int J Commun Based Nurs Midwifery. 2015;3:51.

19. Di Renzo GC, Rosati A, Sarti RD, Cruciani L, Cutuli AM. Does fetal sex affect pregnancy outcome? Gender Med. 2007:4:19-30.

20. Lange S, van Leeuwen P, Geue D, Hatzmann W, Grönemeyer D. Influence of gestational age, heart rate, gender and time of day on fetal heart rate variability. Med Biol Eng Comput. 2005;43:481-486.

21. Pink? Blue? You're pregnant. Now you want to know the sex of your baby 2014 [updated Oct, 2014]. Available from: http://www.babybpm. $\mathrm{com} /$.

22. McKenna D, Ventolini G, Neiger R, Downing C. Gender-related differences in fetal heart rate during first trimester. Fetal Diagn Ther. 2006;21:144-147.

23. Petrie B, Segalowitz SJ. Use of fetal heart rate, other perinatal and maternal factors as predictors of sex. Percept Mot Skills. 1980;50:871-874.

24. Cuestas E, Bas J, Pautasso J. Sex differences in intraventricular hemorrhage rates among very low birth weight newborns. Gender Med. 2009;6:376-382.

25. DuBose TJ, Dickey D, Butschek C, Porter L, Hill LW, Poole EK. Fetal heart rate (FHR) is not an indicator of the baby's sex. J Ultrasound Med. 1988;7:237.

26. Naeye RL, Burt LS, Wright DL, Blanc WA, Tatter D. Neonatal mortality, the male disadvantage. Pediatrics. 1971;48:902-906.

This work is licensed under a Creative Commons Attribution-NonCommercial 3.0 Unported License which allows users to read, copy, distribute and make derivative works for non-commercial purposes from the material, as long as the author of the original work is cited properly. 